\title{
2-Dimensional foot FE models for clinical application in gait analysis
}

\author{
Alessandra Scarton ${ }^{1 *}$, Annamaria Guiotto ${ }^{1}$, Zimi Sawacha', Gabriella Guarneri², Angelo Avogaro², Claudio Cobelli ${ }^{1}$ \\ From 4th Congress of the International Foot and Ankle Biomechanics (i-FAB) Community \\ Busan, Korea. 8-11 April 2014
}

\section{Background}

Foot ulcerations are one of the most common and invalidating complications which affect the diabetic patients $[1,2]$. Several two-dimensional (2D) finite element (FE) models of the foot have been developed in the last decades in order to understand what are the causes and to decrease their progress [3-5].

The aim of this work was to create four 2D FE models of an healthy and of a diabetic neuropathic subject integrating kinematic, kinetic and pressure data and to validate them by means of a comparison between experimental and simulated pressure values. These models could represent a tool for clinical applications in order to prevent the development of the diabetic ulcers.

\section{Methods}

Foot biomechanical analysis was carried out as in [6,7] on 10 healthy (age $58.7 \pm 10$ years, BMI $24.5 \pm 2.6 \mathrm{~kg} / \mathrm{m}^{2}$ ) and 10 diabetic subjects with neuropathy (age 63.2 \pm 6.4 years, BMI $24.3 \pm 2.9 \mathrm{~kg} / \mathrm{m}^{2}$ ). The experimental setup included a $60 \mathrm{~Hz} 6$ cameras stereophotogrammetric system (BTS S.r.l, Padova), 2 force plates (FP4060-10, Bertec Corporation, USA) and 2 plantar pressure systems (Imagortesi, Piacenza). The signals coming from all systems were synchronized as in [6,7].

Four 2D FE models of the foot were developed from MRI images of a healthy and a diabetic subject (Figure 1). The modeled section were chosen as typical areas of ulcers development and according to the position of the marker in the gait analysis protocol: the slice passing through the first and the fifth metatarsal heads, the slice passing through the malleoli, the slice passing through the calcaneus and the second metatarsal head and the

\footnotetext{
* Correspondence: alessandra.scarton@dei.unipd.it

'Department of Information Engineering, University of Padova, Padova, 35131, Italy

Full list of author information is available at the end of the article
}

slice passing through the calcaneus and the first metatarsal head.

The displacements of the markers determined from the gait analysis data for each patient in four instances of the stance phase of gait (initial contact, loading response, midstance and push-off) were used as input for the simulations. The validations of the models have been performed computing the RMSE between the experimental and the simulated plantar pressures in percentage of the experimental peak value.

\section{Results}

Results for the diabetic subjects are shown in Table 1. No significant differences were found between the healthy subjects experimental and simulated pressures.

\section{Conclusions}

Even under the restrictive assumptions of 2D representation, which is inadequate for a complete model of the complex mechanics of the foot, it is possible to run fast computational simulations that provide useful information for the clinicians towards a prevention of plantar ulcer formation.

\section{Authors' details}

${ }^{1}$ Department of Information Engineering, University of Padova, Padova,

35131, Italy. ${ }^{2}$ Department of Clinical Medicine and Metabolic Disease,

University Polyclinic, Padova, 35131, Italy.

Published: 8 April 2014

\section{References}

1. Boulton AJM, Vileikyte L, Ragnarson-Tennvall G, Apelqvist J: The global burden of diabetic foot disease. Lancet 2005, 366:1719-24.

2. Cavanagh P, Erdemir A, Petre M, Owings T, Botek G, Chokhandre S, Bafna R: Biomechanical factors in diabetic foot disease. Journal of Foot and Ankle Research 2008, 1:K4.

3. $W u$ L: Nonlinear finite element analysis for musculoskeletal biomechanics of medial and lateral plantar longitudinal arch of Virtual 

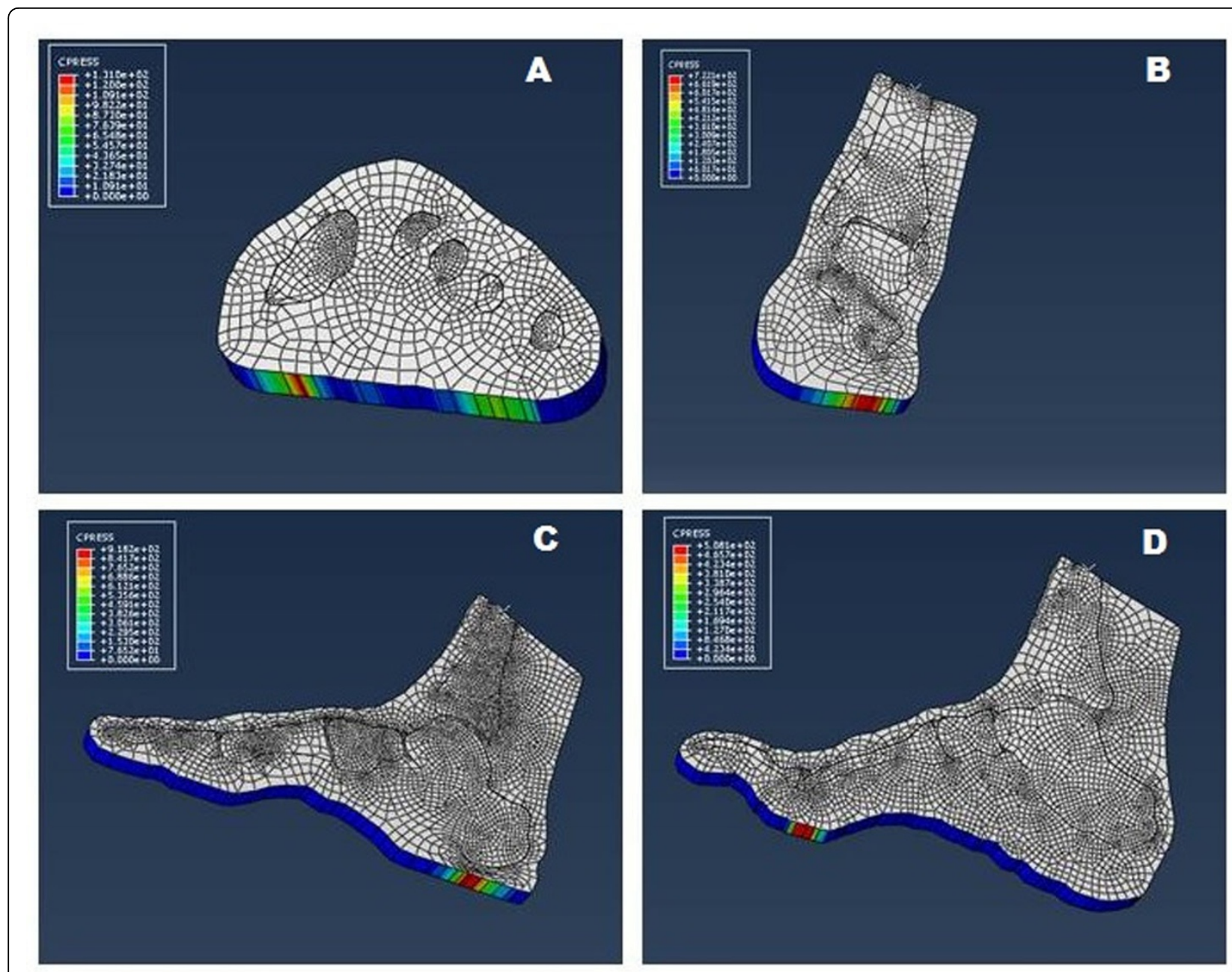

Figure 1 The figure shows the four models developed for the diabetic subject. A) $1^{\text {st }}-5^{\text {th }}$ metatarsal head model; B) Through malleoli model; C) $1^{\text {st }}$ metatarsal -calcaneus model; D) $2^{\text {st }}$ metatarsal -calcaneus model.

Table 1 RMSE between the experimental and the simulated plantar pressures in percentage of the experimental peak value, in four instances of the stance phase of gait and for the four models.

\begin{tabular}{ccccc}
\hline & Initial contact & Loading response & Midstance & Push-off \\
\hline $\mathbf{1}^{\text {st }}$ metatarsal -calcaneus model & 25.08 & 20.43 & 33.26 & 24.69 \\
\hline $\mathbf{2}^{\text {st }}$ metatarsal -calcaneus model & 22.20 & 24.68 & 37.82 & 41.67 \\
\hline $\mathbf{1}^{\text {st }}-\mathbf{5}^{\text {th }}$ metatarsal head model & - & 45.77 & 46.34 & 46.25 \\
\hline Through malleoli model & 35.36 & 42.12 & 46.58 \\
\hline
\end{tabular}

Chinese Human after plantar ligamentous structure failures. Clinical Biomechanics 2007, 22:221-229.

4. Scarton A, Guiotto A, Sawacha Z, Guarneri G, Avogaro A, Cobelli C: Gait analysis driven $2 \mathrm{~d}$ finite element model of the neuropathic hindfoot. Proceedings ISB Natal, Brasil; 2013.

5. Gefen A, Megido-Ravid M, Itzchack Y, Arcan M: Biomechanical analysis of the three-dimensional foot structure during gait: a basic tool for clinical applications. ASME 2000, 122.

6. Sawacha Z, Guarneri G, Cristoferi G, Guiotto A, Avogaro A, Cobelli C: Integrated kinematics-kinetics-plantar pressure data analysis: A useful tool for characterizing diabetic foot biomechanics. Gait \& Posture 2012, 36:20-26.
7. Sawacha Z, Cristoferi G, Guarneri G, Corazza S, Donà G, Denti $P$, Facchinetti A, Avogaro A, Cobelli C: Characterizing multisegment foot kinematics during gait in diabetic foot patients. Journal of NeuroEngineering and Rehabilitation 2009, 6:37.

doi:10.1186/1757-1146-7-S1-A73

Cite this article as: Scarton et al:: 2-Dimensional foot FE models for clinical application in gait analysis. Journal of Foot and Ankle Research 2014 7(Suppl 1):A73. 